\title{
Adição de redutores de pH e doses de glyphosate na dessecação de plantas daninhas ${ }^{1}$
}

\author{
Addition of $\mathrm{pH}$ reducers and rates of glyphosate in the weeds desiccation
}

\author{
Miriam Hiroko Inoue ${ }^{2}$, Roberto Akitoshi Komatsu ${ }^{3}$, Roberto Marins Guerreiro ${ }^{4}$, \\ Rivanildo Dallacort ${ }^{2}$, Diogo Carneiro de Santana ${ }^{5}$, Cleverton Tiago Carneiro de Santana ${ }^{5}$
}

Resumo - Foram conduzidos dois experimentos visando avaliar a influência de redutores de $\mathrm{pH}$ e doses de glyphosate associados à calda de pulverização sobre a dessecação de Euphorbia heterophylla (Amendoim-bravo) e Glycine max (Soja tiguera). Adotou-se o esquema fatorial (4 x $2)+1$, em blocos casualizados com quatro repetições. Foi utilizado um redutor comercial em cada experimento (pH Fós ou Redumax), sendo quatro doses do redutor $(0,50,75$ e $100 \%$ da dose recomendada pelo fabricante) e duas doses de glyphosate (450 e $\left.900 \mathrm{~g} \mathrm{ha}^{-1}\right)$, além de uma testemunha sem aplicação. O controle das plantas daninhas foi avaliado aos 4, 6, 8, 10, 12 e 14 dias após a aplicação (DAA) dos tratamentos, em escala visual de 0 a $100 \%$ de controle. Em ambos os experimentos, na dose de $450 \mathrm{~g} \mathrm{ha}^{-1}$ de glyphosate, a adição de redutores à calda de pulverização proporcionou maior controle das plantas daninhas, em relação ao uso de glyphosate isolado. Não houve diferenças no desempenho proporcionado pelas quantidades de 50,75 e $100 \%$ da dose recomendada dos redutores, em todas as avaliações. Constatou-se ainda que aos 14 DAA, o uso de adjuvante permitiu um controle satisfatório com metade da dose de glyphosate recomendada. Tais resultados evidenciam a possibilidade de redução na dose de glyphosate quando forem utilizados esses adjuvantes.

Palavras-chave: Adjuvantes, doses reduzidas, herbicida, manejo.

\begin{abstract}
Two experiments were conducted to evaluate the influence from the addition of $\mathrm{pH}$ reducers and rates of glyphosate in the spray mix to Euphorbia heterophylla and Glycine max control. The treatments were arranged in a factorial scheme $(4 \times 2)+1$, randomized blocks design and four replications. Each experiment used a $\mathrm{pH}$ reducer ( $\mathrm{pH}$ Fós ou Redumax), four rates of reducer $(0,50,75$ and $100 \%$ of the rate recommended), two rates of glyphosate (450 and $900 \mathrm{~g} \mathrm{ha}^{-1}$ ) and an untreated check. The weed control was evaluated at 4, 6, 8, 10, 12 and 14 days after application (DAA) of treatments, in visual scale from 0 to $100 \%$ of control. In both experiments, $450 \mathrm{~g} \mathrm{ha}^{-1}$ glyphosate, the addition of reducers in the spray mix provided greater weed control in relation to only glyphosate. There was no difference in 50, 75 and $100 \%$ of the rate recommended of reducers. With the use of adjuvant has a satisfactory control with half the recommended rate of glyphosate at 14 DAA. These results demonstrate the possibility of reduced rates of glyphosate when used these adjuvants in the spray mix.
\end{abstract}

\footnotetext{
${ }^{1}$ Recebido para publicação em / / e na forma revisada em / / .

${ }^{2}$ Professor(a) do Departamento de Agronomia da Universidade do Estado de Mato Grosso (UNEMAT), Rodovia MT 358, Km 7, 78300-000, Tangará da Serra, MT, <miriamhinoue@ hotmail.com>;

${ }^{3}$ Professor do Curso de Agronomia da Faculdade Integrado de Campo Mourão;

${ }^{4}$ Eng. Agr. Faculdade Integrado de Campo Mourão;

${ }^{5}$ Alunos do Curso de Agronomia da UNEMAT.
}

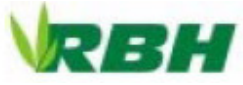


Key-words: Adjuvants, reduced rates, herbicide, management.

\section{Introdução}

O manejo no plantio direto é importante não só para que a cultura inicialmente esteja sem a interferência das plantas daninhas, mas também, pode ser decisivo para determinar o adequado funcionamento dos herbicidas utilizados após a emergência da cultura, pois caso não seja eficiente, podem ocorrer rebrotas e reinfestação na área, com dificuldades à ação de herbicidas utilizados após a semeadura da cultura (Constantin et al., 2007). No entanto, garantir que os herbicidas apresentem eficiência sobre as plantas daninhas tem sido um dos principais desafios da tecnologia de aplicação.

Alguns estudos indicam que a redução no valor do $\mathrm{pH}$ na calda de pulverização tem sido uma prática promissora para melhorar a absorção do herbicida, o que permite reduzir as doses dos produtos e dos custos de produção, mantendo eficiente nível de controle (Carlson \& Burnside, 1984; Azevedo, 2001; Vargas \& Bortolozzo, 2001; Sanchotene et al., 2007). Segundo Sanchotene et al. (2007), herbicidas dissolvidos em condições de baixo $\mathrm{pH}$ podem ser absorvidos com maior facilidade pelas plantas, visto que as moléculas encontram-se na forma não-dissociada.

Para a redução do $\mathrm{pH}$ na calda de pulverização é usado comumente ácido fosfórico, tendo várias marcas comerciais disponibilizadas no mercado (Azevedo, 2001). Uma outra alternativa que visa melhorar a eficiência dos herbicidas é a adição de sulfato de amônia ou uréia na calda de pulverização. Esse processo auxilia a passagem das moléculas na cutícula, fazendo com que o herbicida penetre facilmente na epiderme da folha, além de evitar o escorrimento do produto sobre a folha (Pitelli, 1985).
De acordo com Galli \& Montezuma (2005), o glyphosate apresenta a melhor eficiência com a água a pH 3,5. Costa et al. (2005) também verificaram que a adição de adjuvantes na solução de glyphosate melhorou o espalhamento da gota de pulverização nas superfícies foliares, capacidade atribuída à redução na tensão superficial da solução, aumentando a área de contato da gota com a epiderme da folha.

Apesar dos relatos indicarem que a diminuição do $\mathrm{pH}$ na calda do glyphosate é uma prática positiva, praticamente não há informações concretas referentes à utilização de redutores de $\mathrm{pH}$ visando aumentar a eficiência no controle das plantas daninhas. $\mathrm{O}$ objetivo desse trabalho foi avaliar a influência de redutores de $\mathrm{pH}$ utilizados na calda de pulverização sobre duas doses de glyphosate na dessecação de plantas daninhas.

\section{Material e métodos}

Os experimentos foram conduzidos em Araruna (PR), latitude $24^{\circ} 01^{\prime} 55^{\prime}$ 'S e longitude $52^{\circ} 42^{\prime} 59^{\prime}$ 'W, altitude de $424 \mathrm{~m}$, região centro ocidental do Paraná. A região apresenta temperatura média anual de $26^{\circ} \mathrm{C}$, precipitações pluviométricas anual de $1200 \mathrm{~mm}$ e umidade relativa média do ar de 56\% (COAMO, 2006).

Nos locais em que os experimentos foram conduzidos, coletou-se amostra da água utilizada nas aplicações dos pesticidas para análise das características químicas (Tabela 1). A água foi classificada como mole devido à concentração de $\mathrm{CaCo}_{3} \mathrm{mg} \mathrm{L}^{-1} \mathrm{H}_{2} \mathrm{O}$ (mg por litros de água) ser menor que $50 \mathrm{mg} \mathrm{L}^{-1}$ (Rocha et al., 2004), contendo somente $26,7 \mathrm{mg} \mathrm{L}^{-1}$. 
Tabela 1. Características químicas da amostra de água no local de abastecimento. Araruna, PR, 2006.

\begin{tabular}{lccccccc}
\hline $\mathbf{p H}$ & $\begin{array}{c}\text { Alcalinidade } \\
\mathbf{C a C O}_{\mathbf{3}}\left(\mathbf{m g ~ L}^{-1}\right)\end{array}$ & $\left.\begin{array}{c}\text { Dureza Total } \\
\mathbf{C a C O}_{\mathbf{3}}\left(\mathbf{m g ~ L}^{-1}\right)\end{array}\right)$ & $\begin{array}{c}\text { Bicarbonatos } \\
\mathbf{C a C O}_{\mathbf{3}}\left(\mathbf{m g ~ L}^{-1}\right)\end{array}$ & $\begin{array}{c}\text { Cálcio } \\
\left(\mathbf{m g ~ L}^{-1}\right)\end{array}$ & $\begin{array}{c}\text { Magnésio } \\
\left(\mathbf{m g ~ L}^{-1}\right)\end{array}$ & $\begin{array}{c}\text { Ferro } \\
\left(\mathbf{m g ~ L}^{-1}\right)\end{array}$ & $\begin{array}{c}\text { Alumínio } \\
\left(\mathbf{m g ~ L}^{-1}\right)\end{array}$ \\
\hline 6,87 & 34,0 & 26,7 & 34,0 & 7,01 & 2,24 & 1,02 & 0,167 \\
\hline
\end{tabular}

Fonte: Laboratório Santa Rita, Mamborê, PR.

As áreas experimentais foram cultivadas com soja na safra anterior (2005/06) e encontrava-se em pousio. Praticamente $99 \%$ das áreas estava infestada por duas espécies de plantas: amendoim-bravo (Euphorbia heterophylla) e soja tigüera (Glycine max), com densidade média de 30 e 10 plantas $\mathrm{m}^{-2}$, respectivamente. A aplicação dos tratamentos foi em 12 de maio de 2006, quando ambas as espécies estavam no início de florescimento (Figura 1).

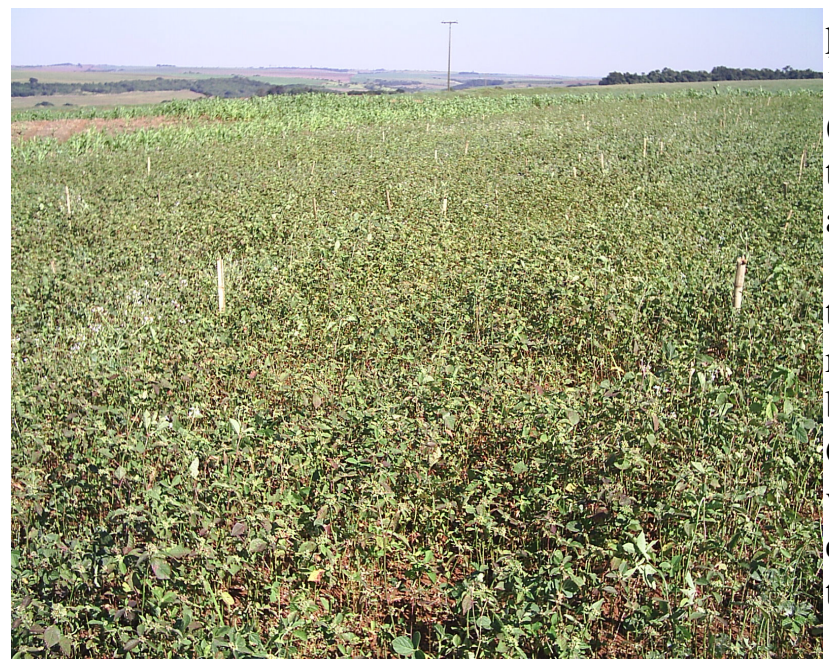

Figura 1. Aspecto geral das áreas em que os experimentos foram desenvolvidos, antes da aplicação dos tratamentos. Araruna, PR, 2006.

Nos experimentos foi adotado o esquema fatorial $(4 \times 2)+1$, em blocos casualizados com quatro repetições, sendo que para cada experimento foi utilizado um redutor de $\mathrm{pH}$ (Tabela 2). Os tratamentos resultaram na combinação de quatro doses do redutor $(0,50$, 75 e $100 \%$ da dose recomendada pelo fabricante) e duas doses de glyphosate (450 e
$900 \mathrm{~g} \mathrm{ha}^{-1}$ ), além de uma testemunha sem herbicida (Tabela 3). A área de cada parcela experimental foi de $30 \mathrm{~m}^{2}(5 \times \mathrm{x} 6 \mathrm{~m})$, totalizando 36 parcelas em cada experimento.

Em ambos os experimentos, as condições climáticas no momento da aplicação foram de $72 \%$ de umidade relativa (UR), $28^{\circ} \mathrm{C}$ de temperatura e solo pouco úmido. As aplicações foram realizadas com pulverizador costal de precisão pressurizado por $\mathrm{CO}_{2}$, equipado com barra de quatro pontas tipo leque XR110.02, pressão de 30 libras pol$^{-2}$, o que proporcionou volume de aplicação de $200 \mathrm{~L} \mathrm{ha}^{-}$ ${ }^{1}$. No período de até 7 dias após aplicação (DAA), as condições climáticas foram de temperatura média de $24^{\circ} \mathrm{C}$, UR de $50 \%$ e sem a ocorrência de chuvas (COAMO, 2006).

As avaliações de controle dos tratamentos sobre as plantas daninhas foram realizadas aos 4, 6, 8, 10, 12 e 14 DAA, com base em escala visual de 0 a $100 \%$ de controle. Os resultados foram submetidos a análise de variância, quando constatada significância estatística, as médias foram comparadas pelo teste de Tukey a 5\% de probabilidade (SAEG, 1997).

\section{Resultados e discussão}

Os dados do experimento 1 , referentes ao controle de plantas daninhas após a aplicação dos diferentes tratamentos, são apresentados na Tabela 4. Independentemente da época de avaliação, verificou-se que o uso de $\mathrm{pH}$ Fós proporcionou os melhores controles das espécies, nos tratamentos que receberam a menor dose de glyphosate $\left(450 \mathrm{~g} \mathrm{ha}^{-1}\right)$. 
Tabela 2. Características dos redutores de pH utilizados nos experimentos. Araruna, PR, 2006.

\begin{tabular}{cclcc}
\hline Experimento & $\begin{array}{c}\text { Nome } \\
\text { Comercial }\end{array}$ & \multicolumn{1}{c}{ Composição Química } & Fabricante & $\begin{array}{c}\text { Dose } \\
(\mathbf{L})^{*}\end{array}$ \\
\hline \multirow{2}{*}{ pH Fós } & $\begin{array}{l}\text { Pentóxido de fósforo }\left(\mathrm{P}_{2} \mathrm{O}_{5}\right) \text { solúvel } \\
\text { em }(\mathrm{CNA}+\text { água })(50 \%) ; \text { Densidade } \\
1,60 ; \mathrm{N} \text { total }(1 \%)\end{array}$ & Quimifol & 0,06 \\
& & $\begin{array}{l}\text { Pentóxido de fósforo } \mathrm{P}_{2} \mathrm{O}_{5} \text { solúvel } \\
\text { em }(\mathrm{CNA}+\text { água) }(30 \%), \text { Densidade } \\
1,42 ;(\text { complexante) } \mathrm{N} \text { total }(3 \%) .\end{array}$ & Forquímica & 0,12 \\
& Redumax & & \\
\hline *Quantidade recomendada pelo fabricante a cada $1000 \mathrm{~L}$ de calda para pulverização. & &
\end{tabular}

Tabela 3. Tratamentos, doses e pH inicial da calda de pulverização utilizados nos dois experimentos. Araruna, PR, 2006.

\begin{tabular}{cccc}
\hline Experimento & Tratamento & Dose $\left(\mathbf{h a}^{-1}\right)$ & pH inicial da calda* \\
\hline & Testemunha & - & - \\
1 & pH Fós + Glyphosate** & $0+450 \mathrm{~g}$ & 6,8 \\
& pH Fós + Glyphosate & $6 \mathrm{~mL}+450 \mathrm{~g}$ & 6,0 \\
& pH Fós + Glyphosate & $9 \mathrm{~mL}+450 \mathrm{~g}$ & 5,0 \\
& pH Fós + Glyphosate & $12 \mathrm{~mL}+450 \mathrm{~g}$ & 3,8 \\
& pH Fós + Glyphosate & $0+900 \mathrm{~g}$ & 6,8 \\
& pH Fós + Glyphosate & $6 \mathrm{~mL}+900 \mathrm{~g}$ & 6,0 \\
& pH Fós + Glyphosate & $9 \mathrm{~mL}+900 \mathrm{~g}$ & 5,0 \\
& pH Fós + Glyphosate & $12 \mathrm{~mL}+900 \mathrm{~g}$ & 3,8 \\
\hline & Testemunha & - & - \\
& Redumax + Glyphosate & $0+450 \mathrm{~g}$ & 6,8 \\
& Redumax + Glyphosate & $12 \mathrm{~mL}+450 \mathrm{~g}$ & 6,0 \\
& Redumax + Glyphosate & $18 \mathrm{~mL}+450 \mathrm{~g}$ & 5,3 \\
& Redumax + Glyphosate & $24 \mathrm{~mL}+450 \mathrm{~g}$ & 4,0 \\
& Redumax + Glyphosate & $0+900 \mathrm{~g}$ & 6,8 \\
& Redumax + Glyphosate & $12 \mathrm{~mL}+900 \mathrm{~g}$ & 6,0 \\
& Redumax + Glyphosate & $18 \mathrm{~mL}+900 \mathrm{~g}$ & 5,3 \\
& Redumax + Glyphosate & $24 \mathrm{~mL}+900 \mathrm{~g}$ & 4,0 \\
\hline
\end{tabular}

*Valor após a adição do redutor, mas antes de acrescentar o glyphosate. Após a adição de glyphosate, os valores na calda variaram entre pH 3,6 e 3,9.**Glyphosate (Foi utilizado o produto comercial Round up Original).

Para a dose $900 \mathrm{~g} \mathrm{ha}^{-1}$, não houve diferença significativa entre os tratamentos químicos, sendo todos esses superiores à testemunha (Tabela 4). Portanto, a utilização do pH Fós na calda de pulverização não influenciou o controle de E. heterophyla e $G$. max na dose recomendada.

Quando em presença de adjuvante, Vargas \& Roman (2006) também observaram melhores resultados no controle de plantas daninhas com a redução de até $50 \%$ na dose 
de herbicidas, comparativamente àquelas sem a adição de adjuvantes. Stougaard (1997) relata que os redutores de $\mathrm{pH}$ são acrescentados à calda de pulverização com o intuito de melhorar a eficiência das pulverizações foliares de herbicidas, reduzir o impacto das interferências ambientais e permitir uma penetração cuticular mais eficiente. Ao analisar os tratamentos nos quais foram adicionados 6,9 e $12 \mathrm{~mL}$ de ha ${ }^{-1}$ do pH Fós, observou-se que não houve diferenças significativas no controle das plantas daninhas, independente da quantidade de glyphosate aplicada (Tabela 4).

Quando em presença de adjuvante, Vargas \& Roman (2006) também observaram melhores resultados no controle de plantas daninhas com a redução de até 50\% na dose de herbicidas, comparativamente àquelas sem a adição de adjuvantes. Stougaard (1997) relata que os redutores de $\mathrm{pH}$ são acrescentados à calda de pulverização com o intuito de melhorar a eficiência das pulverizações foliares de herbicidas, reduzir o impacto das interferências ambientais e permitir uma penetração cuticular mais eficiente. Ao analisar os tratamentos nos quais foram adicionados 6,9 e $12 \mathrm{~mL} \mathrm{de} \mathrm{ha}^{-1}$ do pH Fós, observou-se que não houve diferenças significativas no controle das plantas daninhas, independente da quantidade de glyphosate aplicada (Tabela 4).

Considerando o nível de $80 \%$ de controle, como o mínimo desejável para as condições de campo, observou-se na Tabela 4 que valores superiores a esse foram constatados nos tratamentos químicos aos 12 e 14 DAA, respectivamente para 900 e $450 \mathrm{~g}$ $\mathrm{ha}^{-1}$ de glyphosate. Exceção ocorreu no tratamento em que foi utilizado somente a menor dose de herbicida, com $0 \%$ de controle (Tabela 4). Esses resultados indicam que, quando doses reduzidas de glyphosate forem utilizadas, a adição do pH Fós à calda de pulverização pode proporcionar maior eficiência no controle das plantas daninhas, em relação à mesma dose de glyphosate aplicada isoladamente. Sumner (1997) e Wolf (2000) relatam que a adição de redutores à calda de pulverização pode aumentar o diâmetro das gotas pulverizadas e reduzir o risco potencial de deriva, melhorando o controle de plantas daninhas mesmo em doses reduzidas.

Resultados semelhantes ao experimento 1 também foram constatados no experimento 2, com a utilização do Redumax (Tabela 5). Portanto, todos os tratamentos nos quais se adotou a dose recomendada de glyphosate não apresentaram diferenças significativas nas diferentes épocas avaliadas, independente da quantidade adicionada do Redumax. Dessa forma, evidencia-se que o uso da dose recomendada pelo fabricante (Rodrigues \& Almeida, 2005) pode promover o controle eficiente das plantas daninhas, mesmo sem a utilização de redutor de $\mathrm{pH}$. As doses recomendadas pelos fabricantes para os herbicidas são estabelecidas em quantidades tais que assegurem o controle de inúmeras espécies, com níveis de susceptibilidade diversos e sob condições que possam ser diferentes daquelas consideradas ótimas da ação dos produtos (King \& Oliver, 1992; Klingman et al., 1992). Porém, em condições ambientais favoráveis, as doses dos herbicidas podem ser reduzidas e, ainda assim, controlar eficientemente as plantas daninhas (Devlin et al., 1991).

Até aos 12 DAA, verificou-se que a dose de $900 \mathrm{~g} \mathrm{ha}^{-1}$ glyphosate proporcionou maior controle de E. heterophyla e G. max, em relação à meia dose (Tabela 5). Segundo Hetherington et al. (1998), há a necessidade de uma concentração adequada de moléculas para que o produto possa atingir o sítio de ação da planta daninha e controlá-la eficientemente. 
Tabela 4. Porcentagem de controle de E. heterophylla e G. $\max$ aos 4, 6, 8, 10, 12 e 14 dias após a aplicação de diferentes tratamentos. Experimento 1. Araruna, PR, 2006.

\begin{tabular}{|c|c|c|c|c|c|c|}
\hline \multirow[b]{2}{*}{$\begin{array}{c}\text { Dose de } \\
\text { glyphosate } \\
\left(\mathrm{g} \mathrm{ha}^{-1}\right)\end{array}$} & \multicolumn{5}{|c|}{ Tratamentos } & \multirow[b]{2}{*}{$\begin{array}{l}\text { C. V. } \\
(\%)\end{array}$} \\
\hline & Testemunha & $\begin{array}{c}\text { Glyphosate } \\
\text { (pH 6,85) }\end{array}$ & $\begin{array}{c}\text { pH Fós + } \\
\text { Glyphosate } \\
(\text { pH 6,0) }\end{array}$ & $\begin{array}{c}\text { pH Fós + } \\
\text { Glyphosate } \\
\text { (pH 5,0) }\end{array}$ & $\begin{array}{c}\text { pH Fós + } \\
\text { Glyphosate } \\
\text { (pH 3,8) }\end{array}$ & \\
\hline & \multicolumn{5}{|c|}{ \% de controle aos 4 dias após a aplicação dos tratamentos } & \multirow{3}{*}{14,1} \\
\hline 900 & $0 \mathrm{Ab}$ & $15 \mathrm{Aa}$ & $15 \mathrm{Aa}$ & $15 \mathrm{Aa}$ & $\mathrm{Aa}$ & \\
\hline \multirow[t]{2}{*}{450} & $0 \mathrm{Ab}$ & $0 \mathrm{Bb}$ & $2 \mathrm{Ba}$ & $2 \mathrm{Ba}$ & 2,3 & \\
\hline & & o de controle : & as após a apl & los tratamen & & \multirow{3}{*}{8,7} \\
\hline 900 & $0 \quad \mathrm{Ab}$ & $30 \quad \mathrm{Aa}$ & $35 \mathrm{Aa}$ & $30 \mathrm{Aa}$ & $30 \quad \mathrm{Aa}$ & \\
\hline \multirow[t]{2}{*}{450} & $0 \mathrm{Ab}$ & $0 \mathrm{Bb}$ & $15 \mathrm{Ba}$ & $10 \mathrm{Ba}$ & $\mathrm{Ba}$ & \\
\hline & & de controle : & as após a apl & los tratamen & & \multirow{3}{*}{14,9} \\
\hline 900 & $\begin{array}{ll}0 & \mathrm{Ab}\end{array}$ & $50 \mathrm{Aa}$ & $52 \mathrm{Aa}$ & $50 \quad \mathrm{Aa}$ & $45 \quad \mathrm{Aa}$ & \\
\hline \multirow[t]{2}{*}{450} & $0 \quad \mathrm{Ab}$ & $0 \mathrm{Bb}$ & $25 \mathrm{Ba}$ & $30 \mathrm{Ba}$ & $32 \mathrm{Ba}$ & \\
\hline & & de controle a & ias após a ap & dos tratamen & & \multirow{3}{*}{12,7} \\
\hline 900 & $0 \quad \mathrm{Ab}$ & $75 \mathrm{Aa}$ & $70 \mathrm{Aa}$ & $72 \mathrm{Aa}$ & 75 Аа & \\
\hline \multirow[t]{2}{*}{450} & $0 \quad \mathrm{Ab}$ & $0 \mathrm{Bb}$ & $50 \mathrm{Ba}$ & $45 \mathrm{Ba}$ & $\mathrm{Ba}$ & \\
\hline & & de controle a & ias após a ap & dos tratamen & & \multirow{3}{*}{17,1} \\
\hline 900 & $0 \quad \mathrm{Ab}$ & $95 \quad \mathrm{Aa}$ & $95 \mathrm{Aa}$ & $98 \mathrm{Aa}$ & $100 \mathrm{Aa}$ & \\
\hline \multirow[t]{2}{*}{450} & $0 \quad \mathrm{Ab}$ & $0 \mathrm{Bb}$ & $70 \mathrm{Ba}$ & $75 \mathrm{Ba}$ & $75 \mathrm{Ba}$ & \\
\hline & & de controle a & ias após a ap & dos tratamen & & \multirow{3}{*}{13,3} \\
\hline 900 & $0 \mathrm{Ab}$ & $95 \mathrm{Aa}$ & $100 \mathrm{Aa}$ & $100 \mathrm{Aa}$ & $95 \quad \mathrm{Aa}$ & \\
\hline 450 & $0 \quad \mathrm{Ab}$ & $0 \mathrm{Bb}$ & $90 \mathrm{Aa}$ & $95 \mathrm{Aa}$ & $\mathrm{Aa}$ & \\
\hline
\end{tabular}


Tabela 5. Porcentagem de controle de E. heterophylla e G. $\max$ aos 4, 6, 8, 10, 12 e 14 dias após a aplicação de diferentes tratamentos. Experimento 2. Araruna, PR, 2006.

\begin{tabular}{|c|c|c|c|c|c|c|}
\hline \multirow[b]{2}{*}{$\begin{array}{c}\text { Dose de } \\
\text { glyphosate } \\
\left(\mathrm{g} \mathrm{ha}^{-1}\right)\end{array}$} & \multicolumn{5}{|c|}{ Tratamentos } & \multirow[b]{2}{*}{$\begin{array}{l}\text { C. V. } \\
(\%)\end{array}$} \\
\hline & Testemunha & $\begin{array}{c}\text { Glyphosate } \\
(\mathbf{p H ~ 6 , 8 5 )}\end{array}$ & $\begin{array}{c}\text { Redumax + } \\
\text { Glyphosate } \\
(\text { pH 6,0) }\end{array}$ & $\begin{array}{c}\text { Redumax + } \\
\text { Glyphosate } \\
\text { (pH 5,0) }\end{array}$ & $\begin{array}{c}\text { Redumax + } \\
\text { Glyphosate } \\
\text { (pH 3,8) }\end{array}$ & \\
\hline & \multicolumn{5}{|c|}{ \% de controle aos 4 dias após a aplicação dos tratamentos } & \multirow{3}{*}{10,4} \\
\hline 900 & $0 \mathrm{Ab}$ & $15 \mathrm{Aa}$ & $15 \mathrm{Aa}$ & $15 \mathrm{Aa}$ & $\mathrm{Aa}$ & \\
\hline \multirow[t]{2}{*}{450} & $0 \mathrm{Ab}$ & $0 \mathrm{Bb}$ & $2 \mathrm{Ba}$ & $2,3 \mathrm{Ba}$ & $\mathrm{Ba}$ & \\
\hline & & o de controle : & as após a apli & los tratamen & & \multirow{3}{*}{11,3} \\
\hline 900 & $0 \quad \mathrm{Ab}$ & $30 \quad \mathrm{Aa}$ & 28 Aa & $30 \quad \mathrm{Aa}$ & $28 \quad \mathrm{Aa}$ & \\
\hline \multirow[t]{2}{*}{450} & $0 \mathrm{Ab}$ & $0 \mathrm{Bb}$ & $18 \mathrm{Ba}$ & $15 \mathrm{Ba}$ & $18 \mathrm{Ba}$ & \\
\hline & & de controle : & as após a apli & los tratamen & & \multirow{3}{*}{6,1} \\
\hline 900 & $0 \quad \mathrm{Ab}$ & $50 \mathrm{Aa}$ & $50 \mathrm{Aa}$ & $45 \quad \mathrm{Aa}$ & $\mathrm{Aa}$ & \\
\hline \multirow[t]{2}{*}{450} & $0 \quad \mathrm{Ab}$ & $0 \mathrm{Bb}$ & $30 \mathrm{Ba}$ & $32 \mathrm{Ba}$ & $30 \mathrm{Ba}$ & \\
\hline & & de controle a & ias após a apl & dos tratamen & & \multirow{3}{*}{15,1} \\
\hline 900 & $\begin{array}{ll}0 & \mathrm{Ab}\end{array}$ & $75 \quad \mathrm{Aa}$ & 70 Aa & $75 \quad \mathrm{Aa}$ & $70 \quad \mathrm{Aa}$ & \\
\hline \multirow[t]{2}{*}{450} & $0 \quad \mathrm{Ab}$ & $0 \mathrm{Bb}$ & $52 \mathrm{Ba}$ & $50 \mathrm{Ba}$ & $52 \mathrm{Ba}$ & \\
\hline & & de controle a & ias após a apl & dos tratamen & & \multirow{3}{*}{13,7} \\
\hline 900 & $0 \quad \mathrm{Ab}$ & $95 \mathrm{Aa}$ & $95 \mathrm{Aa}$ & 100 Aa & $95 \mathrm{Aa}$ & \\
\hline \multirow[t]{2}{*}{450} & $0 \quad \mathrm{Ab}$ & $0 \mathrm{Bb}$ & $70 \mathrm{Ba}$ & $75 \mathrm{Ba}$ & $70 \mathrm{Ba}$ & \\
\hline & & de controle a & ias após a apl & dos tratamen & & \multirow{3}{*}{7,4} \\
\hline 900 & $0 \quad \mathrm{Ab}$ & $95 \mathrm{Aa}$ & 98 Aa & $100 \mathrm{Aa}$ & $98 \quad \mathrm{Aa}$ & \\
\hline 450 & $0 \quad \mathrm{Ab}$ & $0 \quad \mathrm{Bb}$ & $90 \mathrm{Aa}$ & $100 \mathrm{Aa}$ & $90 \mathrm{Aa}$ & \\
\hline
\end{tabular}


Em ambas as doses de glyphosate, não foi constatada diferença na eficiência de controle nos tratamentos aos 14 DAA com a adição do Redumax à calda de pulverização (Tabela 5). A partir desses dados, infere-se que é possível reduzir em até 50\% a dose de glyphosate com o uso do Redumax, mantendo a mesma eficiência no controle. Com o intuito de avaliar o desempenho do glyphosate em mistura com adjuvantes, Pedrinho Junior et al. (2002) verificaram que as adições de uréia e sulfato de amônio potencializaram o efeito do herbicida em todas as avaliações visando a dessecação das plantas daninhas. No entanto, a adoção em maior escala da aplicação de doses reduzidas de herbicidas não tem evoluído devido ao risco de que as plantas daninhas poderiam sobreviver, produzir sementes para os anos seguintes $\mathrm{e}$ incrementar o banco de sementes no solo (Jones \& Medd, 2000).

Outro aspecto importante foi verificado na Tabela 5 , em que a quantidade do Redumax adicionada à calda de pulverização $\left(12,18\right.$ e $24 \mathrm{~mL}$ de ha $\left.{ }^{-1}\right)$ não influenciou no controle de plantas daninhas. Por outro lado, considerando a dose de $450 \mathrm{~g}$ $\mathrm{ha}^{-1}$ glyphosate, os tratamentos com o Redumax foram superiores em comparação ao uso de glyphosate isolado (Tabela 5). Alguns trabalhos (Pitelli, 1985; Vargas \& Roman, 2006) indicam que o nitrogênio presente na composição dos redutores de $\mathrm{pH}$ pode favorecer a absorção da calda pela planta melhorando o desempenho do herbicida.

Nos dois experimentos, o controle das plantas daninhas foi crescente nos períodos após a aplicação dos tratamentos (Tabelas 4 e 5), demonstrando que o processo é lento até que ocorra a morte da planta. Isto se deve provavelmente ao fato do glyphosate ser um herbicida aplicado na parte aérea das plantas, cuja eficácia é dependente de processos como a retenção da molécula na superfície foliar, da

absorção foliar e da translocação das moléculas via floema para chegar ao sítio de ação na planta (Hetherington et al., 1998). Outros fatores também podem influenciar a eficácia do produto para controle das plantas daninhas, como a temperatura, a luminosidade, a umidade do solo, a umidade relativa do ar e a ocorrência de geadas (Baird, 1971; Fawcett \& Davis, 1976).

Ambos os redutores de $\mathrm{pH}$ avaliados nesse trabalho obtiveram resultados semelhantes. Nos tratamentos em que foram utilizadas doses reduzidas de glyphosate, o controle foi significativamente favorecido com a adição do pH Fós e do Redumax (Tabelas 4 e 5). Segundo Azevedo (2001), as gotas de pulverização emulsionadas com os redutores de $\mathrm{pH}$ tendem a evaporar de forma lenta e atingem seu alvo mais facilmente.

Outros estudos mostram que a adição de surfactantes à calda aumenta a hidratação da cutícula, incrementando a difusão do glyphosate através desta e, consequientemente, aumenta a absorção foliar (Ruiter \& Mainen, 1998). Aliado a esses fatores, quando os herbicidas são dissolvidos em condições de baixo $\mathrm{pH}$, a maioria das moléculas se encontra na forma nãodissociada, o que proporciona que sejam absorvidas com maior facilidades pelas plantas (Wanamarta \& Penner, 1989).

\section{Conclusões}

Considerando que o glyphosate é o herbicida de maior volume de vendas no mundo, com intenso uso por quase três décadas (Heap, 2008), a utilização deste produto em doses reduzidas poderá contribuir para minimizar a contaminação ambiental.

\section{Referências}


AZEVEDO, L. S. Proteção integrada de plantas com fungicidas. São Paulo: EMOPI. 2001, 230p.

BAIRD, D. D. Introduction of a new broad spectrum postemergent herbicide class with utility for herbaceous perennial weed control. Proc. N. Cent. Weed Contr. Conf., v.26, p.64-68, 1971.

CARLSON, K. L.; BURNSIDE, O. C. Comparative phytotoxicity of Glyphosate, SC-0224, SC-0545, and HOE-00661. Weed Sci., v.32, p.841-844, 1984.

COAMO - Agroindustrial Cooperativa. Dados da Estação Meteorológica da COAMO. 2006.

CONSTANTIN, J.; OLIVEIRA JR., R. S.; CAVALIERI, S. D.; ARANTES, J. G. Z.; ALONSO, D. G.; ROSO, A. C.; COSTA, J. M. Interação entre sistemas de manejo e controle de plantas daninhas em pósemergência afetando o desenvolvimento e a produtividade do milho. Planta Daninha, v.25, p.513-520, 2007.

COSTA, N. V.; MARTINS, D.; RODELLA, R. A.; COSTA, L. D. N. C. pH foliar e deposição de gotas de pulverização em plantas daninhas aquáticas: Brachiaria mutica, Brachiaria subquadripara e Panicum repens. Planta Daninha, v.23, p.295-304, 2005.

DEVLIN, D. L.; LONG, J. H.; MADDUX, L. D. Using reduced rates of post-emergence herbicides and soybeans (Glycyne max). Weed Tech., v.5, p.834-840, 1991.

FAWCETT, R. S.; DAVIS, H. Effect of environment on glyphosate activity in quackgrass. Proc. N. Cent. Weed Contr. Conf., v.31, p.159-160, 1976.

GAlli, B. J. A.; MONTEZUMA, C. M. Alguns aspectos da utilização do herbicida glyphosate na agricultura. Monsanto do Brasil, 2005. 60p.

HEAP, I. 2008 International Survey of Herbicide Resistant Weeds. Online. Internet. 27 de Julho de 2008. Disponível em www.weedscience.org.

HETHERINGTON P. R.; REYNOLDS T. L.; MARSHALL, G.; KIRKWOOD, R. C. The absorption, translocation and distribution of the herbicide Glyphosate in maize expressing the CP-4 transgene. J. Exp. Botany, v.50, p.1567-1576, 1998.

JONES, R. E.; MEDD, R. W. Economic thresholds and the case for longer term approaches to population management of weeds. Weed Tech., v.14, p.337- 350, 2000.

KING, C. A.; OLIVER, L. R. Application rate and timing acifluorfen, bentazon, chlorimuron, and imazaquin. Weed Tech., v.6, p.526-534, 1992.

KLINGMAN, T. E.; KING, C. A.; OLIVER, L. R. Effect of application rate, weed species, and weed stage of growth on imazethapyr activity. Weed Sci., v.40, p.227-232, 1992.

PEDRINHO JUNIOR, A. F. F.; PIVA, F. M.; MARTINI, G.; FELICI, G. V.; DURIGAN, J. C. Influência da chuva na eficácia do glyphosate em mistura com adjuvantes na dessecação de plantas daninhas. Planta Daninha, v.20, p.263-271, 2002. 
PITELLI, R. A. Interferência das plantas daninhas em culturas agrícolas. Inf. Agropec., n.11, 1985.

ROCHA, J. C.; ROSA, A. H.; CARDOSO, A. A. Introdução à química ambiental. Porto Alegre: Bookman, 2004.

RODRIGUES, B. N.; ALMEIDA, F. L. S. Guia de herbicidas, 5.ed. Londrina: IAPAR, 2005. 592p.

RUITER, H.; MAINEN, E. Influence of water stress and surfactant on the efficacy, absorption, and translocation of glyphosate. Weed Sci., v.46, p.289-296, 1998.

SAEG - Sistema para Análises Estatísticas: versão 7.0. Viçosa: Fundação Arthur Bernardes, 1997.

SANCHOTENE, D. M.; DORNELLES, S. H. B.; DEBORTOLI, M. P.; CAPITANIO, JR.; MEZZOMO, R. F.; GONÇALVES, R. A. Influência de sais e do $\mathrm{pH}$ da água na eficiência de imazethapyr + imazapic no controle de arroz-vermelho. Planta Daninha, v.25, p.415-419, 2007.

STOUGAARD, R. M. Adjuvant combinations with quizalofop for wild oat (Avena fatua) control in pepermit (Mentha piperita). Weed Tech., v.11, p.45-50, 1997.

SUMNER, P. E. Reducing spray drift. Georgia: University of Georgia, 1997, 11p.

VARGAS, L.; BORTOLOZZO, A. R. Adição de adjuvantes para superar os efeitos negativos da qualidade da água sobre o glyphosate. Factuciência, v.1, p.19-26, 2001.
VARGAS, L.; ROMAN, E. S. Conceitos e aplicações dos adjuvantes. Passo Fundo: Embrapa Trigo, 2006. 10p.

WANAMARTA, G.; PENNER D. Foliar absorption of herbicides. Weed Sci., v.4, p.215-232, 1989.

WOLF, R. E. Strategies to reduce spray drift. Kansas: Kansas State University, 2000. $4 \mathrm{p}$. 